\title{
Subacute carbon monoxide poisoning presenting as vertigo and fluctuating low frequency hearing loss
}

\author{
Bethany Seale*, Churunal Hari \# \\ *Keele University Medical School, " Royal Shrewsbury Hospital
}

\section{INTRODUCTION}

Up to 25000 UK residents are exposed to small amounts of carbon monoxide (CO) annually ${ }^{1}$. The most common source of carbon monoxide poisoning in domestic setting is a faulty heating system. Symptoms of chronic exposure to $\mathrm{CO}$ are vague and nonspecific and include dizziness and hearing loss².

\section{CASE REPORT}

- A 38-year-old lady presented to ENT with a four-month history of dizziness and hearing loss.

- She reported intermittent hearing loss, mainly on the right side but denied the presence of tinnitus.

- She also suffered with heaviness and pressure sensation in the head. The was no nausea or vomiting.

- Audiogram revealed unilateral sensorineural hearing loss (SNHL), with thresholds between 30 and 40 decibels (Figure 1).

- All other investigations including MRI brain were normal.

- 6 months later, she reported leakage of carbon monoxide from her gas fire identified during a routine safety check.

- Her symptoms fully resolved after disconnecting the faulty gas fire and her audiograms returned to normal.

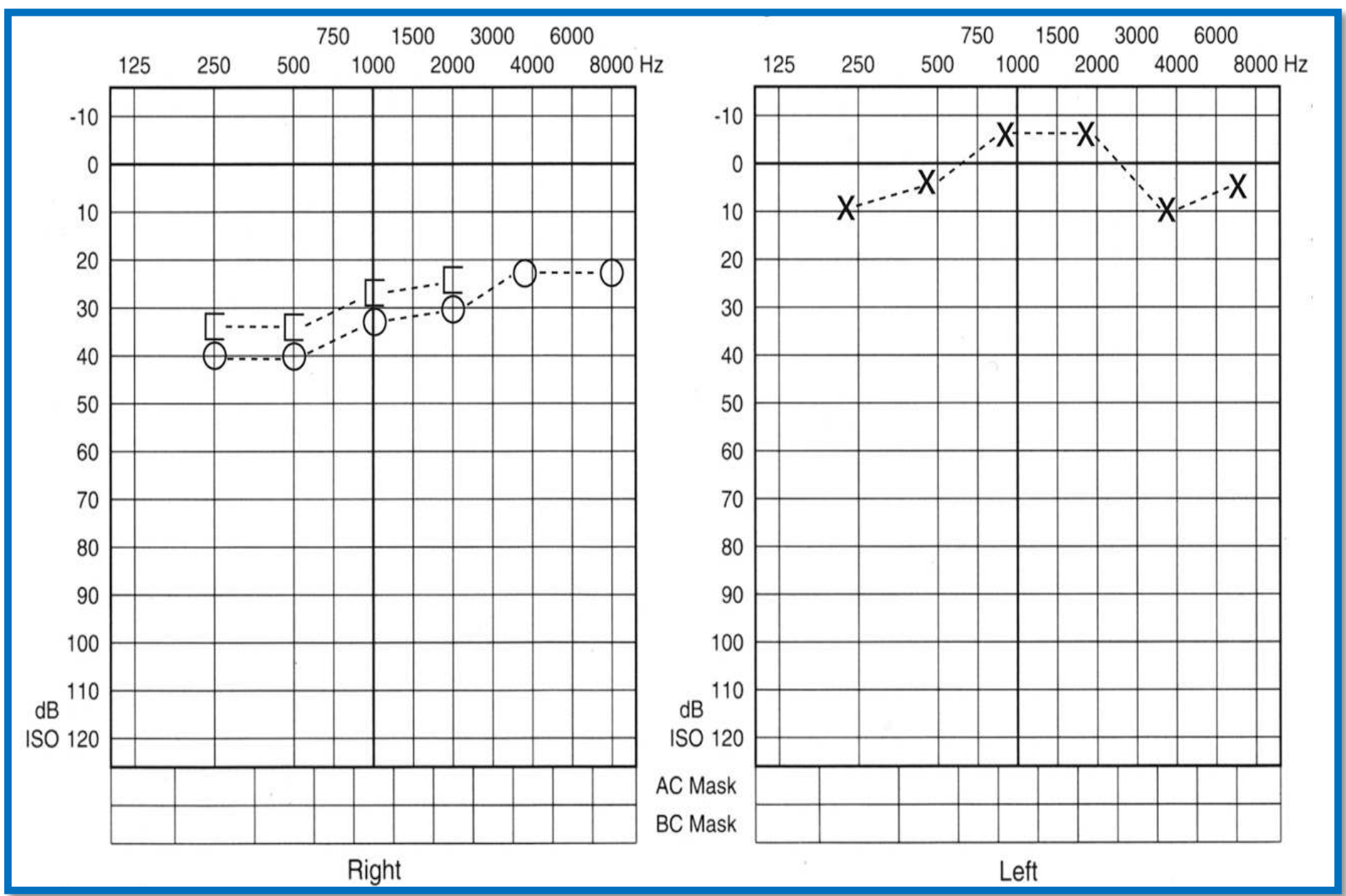

Figure 1: Audiogram demonstrating low frequency hearing loss in the right ear.

\section{DISSCUSSION}

- SNHL in chronic $\mathrm{CO}$ poisoning is well documented ${ }^{3}$.

- Unilateral hearing loss is rare and in association with dizziness can be confused with diagnosis of Meniere's disease.

- Careful detailed history is key in differential diagnosis of dizziness.

- Rare causes like CO poisoning have to be considered in unexplained hearing loss and dizziness.

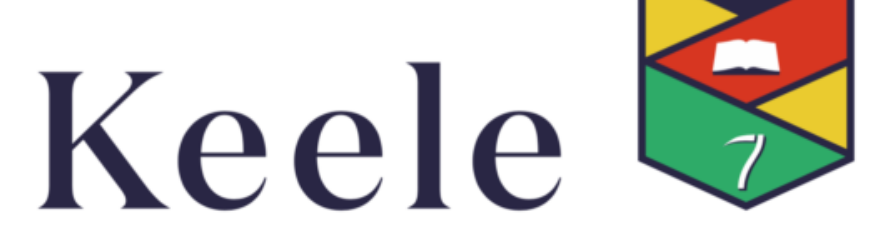

U N I VER S I T Y 